\title{
Impulsive nanofluid flow along a vertical stretching cone
}

\author{
Sami M.S. Ahamed ${ }^{1}$, Sabyasachi Mondal ${ }^{2 *}$, Precious Sibanda ${ }^{1}$ \\ ${ }^{1}$ School of Mathematics, Statistics and Computer Science, University of KwaZulu-Natal Private \\ Bag X01 Scottsville 3209, South Africa \\ ${ }^{2}$ Department of Mathematics, Amity University, Kolkata, Newtown-700135, West Bengal, India
}

Email: sabya.mondal.2007@gmail.com

\begin{abstract}
We study the unsteady convective flow of two water-based nanofluids containing Copper and Titanium oxide along a vertical stretching or shrinking cone with viscous dissipation and internal heat generation. The problem is transformed to two-dimensional flow over a cone using Mangler's transformation. The coupled nonlinear conservation equations are solved numerically using the spectral local linearization method. We present an analysis of how some physical parameters affect the flow structure, the heat and mass transfer rates and the fluid properties. The accuracy of the results is determined by comparison with previously published studies, for some limiting cases.
\end{abstract}

Keywords: Chemical Reaction, Nanofluid Flow, Stretching or Shrinking Cone, Spectral Local Linearization Method.

\section{INTRODUCTION}

Common fluids such as water, ethylene glycol and oil have low heat transfer characteristic owing to their poor thermal conductivities. It is now understood that the thermophysical properties of these fluids can be significantly enhanced by suspending nano-sized metallic particles such as Aluminum, titanium, Gold, Copper, Iron or their oxides, resulting in what is commonly called a nanofluid, see Choi and Eastman [1].

During the last several years many authors have studied the boundary layer flow of nanofluid fluids through different geometries and with different conditions. Examples include Kameswaran et al. [2] who studied hydromagnetic nanofluid flow due to a stretching or shrinking sheet and Kameswaran et al. [3] who found solutions for the equations for the stagnation-point flow of a nanofluid over a stretching surface. The steady nanofluid boundary layer flow along a vertical cone in a porous medium was investigated by Fauzi et al. [4]. Boutra et al. [5] studied free convection enhancement within a nanofluid' filled enclosure with square heaters and Ambethkar and Kumar [6] examined solutions of 2-D unsteady incompressible flow with heat transfer in a driven square cavity sing streamfunction-vorticity formulation. Cheng [7] discussed natural convection in boundary layer flow over a truncated cone embedded in a porous medium. Chamkha et al. [8] considered the problem of steady, laminar, mixed convection boundary-layer flow over a vertical cone embedded in a porous medium with thermal radiation while Nadeem and Saleem [9] investigated unsteady nanofluid flow in a rotating cone subject to an applied magnetic field. In this study we investigate the flow of a nanofluid along a vertical stretching cone. Early studies of boundary layer over a coneshaped surface include those of Heiring and Grosh [10] who studied natural convection along a non-isothermal cone. Tien and Tsuji [11] gave a theoretical analysis of the forced convection flow due to a rotating cone. Koh and Price [12] investigated heat transfer past a rotating cone. Convection in gas flow over an isothermal vertical cone was studied by Takhar et al. [13]. Turkyilmazoglu [14] presented analytical a solution of the equations for steady laminar flow of a Newtonian fluid over a rotating cone. Lately, several researchers such as Sivaraj and Kumar [15], Srinivasa et al. [16] and Roy et al. [17] have presented studies focussing on the flow and heat transfer to or from cone shaped bodies under different assumed conditions. Boundary layer studies of stretching surfaces have a rich history starting with the work of Crane [18]. More recent studies include Mahapatra et al. [19], Salem and abd El-Aziz [20] and Noor et al. [21]. The interest in boundary layer stretching sheet flow problems has partly been driven by industrial processes where such flows occur or may apply. Often cited examples include extrusion processes, the manufacture of plastic products, polymers and rubber sheets, wire and fibre coating, glass and optical fibre production, hot rolling production, metal spinning, food processing and many others.

Notwithstanding the number of studies on both stretching and cone-shaped bodies so far, we study here nanofluid along a vertical stretching or shrinking cone with dissipative heat loss and heat generation. The base fluid is water containing a 
stable suspension of $\mathrm{Cu}$ and $\mathrm{TiO}_{2}$ nanoparticles. The axisymmetric flow problem is transformed to a twodimensional problem and the equations are solved numerically using a spectral local linearization method (SLLM). We give an analysis of the impact of changes in flow parameters on the skin friction, heat transfer and the fluid properties. To validate the results, we give a comparison with the earlier work of Kameswaran et al. [3], Wang [22], Jafar et al. [23].

\section{MATHEMATICAL FORMULATION}

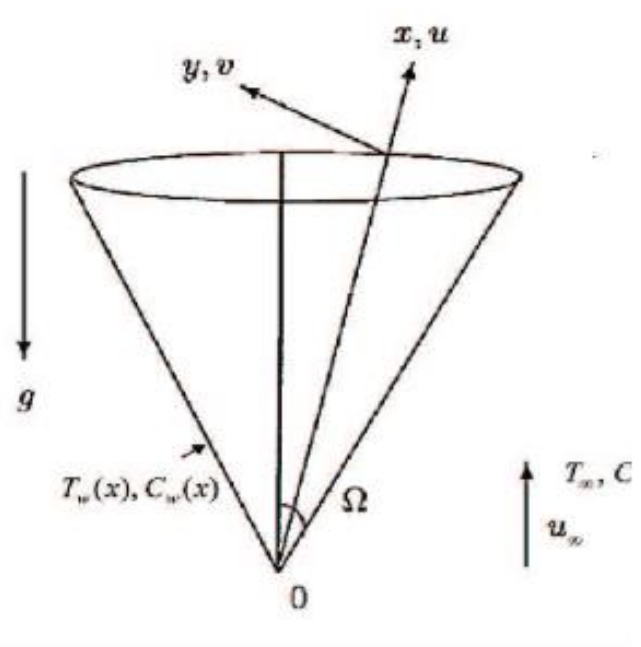

Figure 1. Geometry and the coordinate system

We consider a two-dimensional unsteady boundary layer flow of an incompressible viscous nanofluid along a vertical stretching or shrinking cone embedded in a porous medium. The coordinate system and the physical model are shown in Fig. 1. The cone stretches or shrinks with velocity $U_{0}=u_{0} x^{m / 3}$ where $m$ is an exponent. Mangler's transformation is used (see Schlichting, [24]) to reduce the axisymmetric system to a two-dimensional problem. Then equations can be written as,

$\frac{\partial u}{\partial x}+\frac{\partial v}{\partial y}=0$

$\frac{\partial u}{\partial t}+u \frac{\partial u}{\partial x}+v \frac{\partial u}{\partial y}=-\frac{1}{\rho_{n f}} \frac{\partial p}{\partial x}+v_{n f}\left(\frac{\partial^{2} u}{\partial y^{2}}\right)+\frac{(\rho \beta)_{n f}}{\rho_{n f}}\left(T-T_{\infty}\right) g \cos \Omega-\frac{\sigma B_{0}^{2}}{\rho_{n f}} u$

$\frac{\partial T}{\partial t}+u \frac{\partial T}{\partial x}+v \frac{\partial T}{\partial y}=\alpha_{n f} \frac{\partial^{2} T}{\partial y^{2}}+\frac{Q}{\left(\rho c_{p}\right)_{n f}}\left(T-T_{\infty}\right)+\frac{\mu_{n f}}{\left(\rho c_{p}\right)_{n f}}\left(\frac{\partial u}{\partial y}\right)^{2}$

$\frac{\partial C}{\partial t}+u \frac{\partial C}{\partial x}+v \frac{\partial C}{\partial y}=D_{\mathrm{m}} \frac{\partial^{2} C}{\partial y^{2}}-R\left(C-C_{\infty}\right)$

The boudary conditions are given by

$u=U_{0}=u_{0} x^{m / 3}, v=0, T=T_{\mathrm{w}}, C=C_{\mathrm{w}}$, at $y=0$ and $t>0$,

$u \rightarrow U_{\infty}=u_{\infty} x^{m / 3}, T \rightarrow T_{\infty}, C \rightarrow C_{\infty}$, as $y \rightarrow \infty$ and $t>0$.

subject to the initial conditions

$u=v=0, T=T_{\infty}, C=C \infty$ for $t<0$

The parameters are the effective dynamic viscosity $\mu_{n f}$, the kinematic viscosity $v_{n f}$, the thermal diffusivity $\alpha_{n f}$, the heat capacity $\left(\rho c_{p}\right)_{n f}$, the density $\rho_{n f}$, the thermal expansion coefficient $(\rho \beta)_{n f}$ and the thermal conductivity $k_{n f}$ of nanofluid, which are given by (see Oztop and Abu-Nada [25]),

$$
\begin{aligned}
& \mu_{n f}=\frac{\mu_{f}}{(1-\phi)^{2.5}}, ? v_{n f}=\frac{\mu_{n f}}{\rho_{n f}}, ? \alpha_{n f}=\frac{k_{n f}}{\left(\rho c_{p}\right)_{n f}}, \\
& \left(\rho c_{p}\right)_{n f}=(1-\phi)\left(\rho c_{p}\right)_{f}+\phi\left(\rho c_{p}\right)_{s}, ? \rho_{n f}=(1-\phi) \rho_{f}+\phi \rho_{s}, \\
& (\rho \beta)_{n f}=(1-\phi)(\rho \beta)_{f}+\phi(\rho \beta)_{s}, \frac{k_{n f}}{k_{f}}=\frac{\left(k_{s}+2 k_{f}\right)-2 \phi\left(k_{f}-k_{s}\right)}{\left(k_{s}+2 k_{f}\right)+\phi\left(k_{f}-k_{s}\right)} .
\end{aligned}
$$

We introduce the following transformations,

$$
\begin{aligned}
& \eta=\sqrt{\frac{U_{\infty}}{v_{f} x \xi}} y, f(\eta, \xi)=\frac{\psi}{\sqrt{v_{f} U_{\infty} x \xi}}, ? \\
& \xi=1-e^{-\tau}, \mathcal{Z}=\frac{U_{\infty}}{x} t, \mathscr{O}(\eta, \xi)=\frac{T-T_{\infty}}{T_{\mathrm{w}}-T_{\infty}}, \\
& \Phi(\eta, \xi)=\frac{C-C_{\infty}}{C_{\mathrm{w}}-C_{\infty}}, T_{\mathrm{w}}=T_{\infty}+T_{0} x^{\frac{(2 m-3)}{3}}, ? \\
& C_{\mathrm{w}}=C_{\infty}+C_{0} x^{\frac{(2 m-3)}{3}},
\end{aligned}
$$

And the stream function $\psi$ is chosen such that

$\mathrm{u}=\partial \psi / \partial y, \mathrm{v}=-\partial \psi / \partial x$

Substituting the transformations (7) into Eqs. (1) - (4), the (1) is automatically satisfied and Eqs. (2)-(4) reduce to

$$
\begin{aligned}
& f^{\prime \prime \prime}+\phi_{1}\left\{\frac{1}{2}(1-\xi) \eta f^{\prime \prime}+f_{1} f f^{\prime \prime}+\xi\left[\left(\frac{m}{3}\right)\left(1-\left(f^{\prime}\right)^{2}\right)+\frac{\mathrm{Mn}^{2}}{\phi_{2}}\left(1-f^{\prime}\right)+\frac{\phi_{3}}{\phi_{2}} \lambda \theta\right]\right\} \\
& =\phi_{1} \xi(1-\xi)\left\{f_{2} f^{\prime \prime} \frac{\partial f}{\partial \xi}+\left(1-f_{2} f^{\prime}\right) \frac{\partial f^{\prime}}{\partial \xi}\right\} \\
& \theta^{\prime \prime}+\frac{k_{f}}{k_{n f}} \operatorname{Pr}_{4}\left\{\frac{1}{2}(1-\xi) \eta \theta^{\prime}+f_{1} f \theta^{\prime}-\xi\left[\left(\frac{2 m-3}{3}\right) f^{\prime} \theta-\frac{\delta}{\phi_{4}} \theta\right]+\frac{E c}{\phi_{5}} f^{\prime \prime 2}\right\} ? \\
& =\frac{k_{f}}{k_{n f}} \operatorname{Pr}_{4} \xi(1-\xi)\left\{f_{2} \theta^{\prime} \frac{\partial f}{\partial \xi}+\left(1-f_{2} f^{\prime}\right) \frac{\partial \theta}{\partial \xi}\right\} \\
& \Phi^{\prime \prime}+\operatorname{Sc}\left\{\frac{1}{2}(1-\xi) \eta \Phi^{\prime}+f_{1} f \Phi^{\prime}-\xi\left[\left(\frac{2 m-3}{3}\right) f^{\prime} \Phi+\gamma \Phi\right]\right\} \\
& =S c \xi(1-\xi)\left\{f_{2} \Phi^{\prime} \frac{\partial f}{\partial \xi}+\left(1-f_{2} f^{\prime}\right) \frac{\partial \Phi}{\partial \xi}\right\}
\end{aligned}
$$

The boundary conditions in Eq. (5) are transformed to

$f=0, f^{\prime}=\varepsilon, \theta=\Phi=1$ at $\eta=0,1 \geq \xi \geq 0$,

$f^{\prime}=1, \theta=\Phi=0$ as $\eta \rightarrow \infty, 1 \geq \xi \geq 0$,

where

$$
\begin{aligned}
& f_{1}=\xi\left(\frac{m+3}{6}\right)-\left(\frac{m-3}{6}\right)(1-\xi) \log (1-\xi), ? f_{2}=\left(\frac{m-3}{3}\right) \log (1-\xi), \\
& \phi_{1}=(1-\phi)^{2.5}\left[1-\phi+\phi\left(\frac{\rho_{s}}{\rho_{f}}\right)\right], ? \phi_{2}=\left[1-\phi+\phi \frac{\rho_{s}}{\rho_{f}}\right], ? \phi_{3}=\left[1-\phi+\phi \frac{(\rho \beta)_{s}}{(\rho \beta)_{f}}\right], \\
& \phi_{4}=\left[1-\phi+\phi \frac{\left(\rho c_{p}\right)_{s}}{\left(\rho c_{p}\right)_{f}}\right], ? \phi_{5}=(1-\phi)^{2.5}\left[1-\phi+\phi \frac{\left(\rho c_{p}\right)_{s}}{\left(\rho c_{p}\right)_{f}}\right]
\end{aligned}
$$


In the above equations, the prime denotes differentiation with respect to $\eta$. The parameters are defined as

$$
\begin{aligned}
& M n=\sqrt{\frac{\sigma B_{0}^{2} x}{\rho_{f} U_{\infty}}}, ? \lambda=\frac{G r_{x}}{\operatorname{Re}_{x}^{2}}, ? G r_{x}=\frac{g \beta_{f}\left(T_{\mathrm{w}}-T_{\infty}\right) x^{3} \cos \Omega}{v_{f}^{2}}, ? \\
& \operatorname{Re}_{x}=\frac{U_{\infty} x}{v_{f}}, \operatorname{Pr}=\frac{v_{f}\left(\rho c_{p}\right)_{f}}{k_{f}}, ? \delta=\frac{Q x}{\left(\rho c_{p}\right)_{f} U_{\infty}}, ? \\
& E c=\frac{U_{\infty}^{2}}{\left(c_{p}\right)_{f}\left(T_{\mathrm{w}}-T_{\infty}\right)}, ? S c=\frac{v_{f}}{D_{m}}, ? \gamma=\frac{R x}{U_{\infty}}, ? \varepsilon=\frac{u_{0}}{u_{\infty}} \text {. }
\end{aligned}
$$

where Rex is the local Reynolds number and Grx is the local Grashof number (see Gangadhar et al. [26], Mahdy [27]). It must be noted that $\lambda>0$ corresponds to the case of buoyancy assisting the flow while $\lambda<0$ corresponds to buoyancy opposing the flow and $\lambda=0$ suggests pure forced convection. When $\xi=0$ and $\phi=0$ (regular fluid), Eq. (9) reduces to the ordinary differential equation,

$f^{\prime \prime \prime}+1 / 2 \eta f^{\prime \prime}=0$,

with boundary conditions (when $\varepsilon=0$ ) are

$f(0,0)=0, f^{\prime}(0,0)=0, f^{\prime}(1,0)=1$

In studies of this nature, we also often interested in the skin friction coefficient $C f x$, the Nusselt number $N u x$ and the Sherwood number Shx. These defined as

$C_{f x}=2 \tau_{\mathrm{w}} / \rho_{f} U_{\infty}^{2}, N u_{x}=x q_{\mathrm{w}} / k_{f}\left(T_{\mathrm{w}}-T_{\infty}\right)$,

$S h_{x}=x q_{\mathrm{m}} / D_{\mathrm{m}}\left(C_{\mathrm{w}}-C_{\infty}\right)$.

where $\tau_{\mathrm{w}}$ is the shear stress at the cone surface, $q_{\mathrm{w}}$ and $q_{\mathrm{m}}$ are the heat and mass flux from the cone surface, respectively,

$\tau_{w}=\mu_{n f}\left(\frac{\partial u}{\partial y}\right)_{y=0}, ? q_{w}=-k_{n f}\left(\frac{\partial T}{\partial y}\right)_{y=0}, q_{m}=-D_{m}\left(\frac{\partial C}{\partial y}\right)_{y=0}$

and substituting (6) into (16) and (17), we get

$$
\begin{aligned}
& \frac{1}{2} \sqrt{R e_{x}} C_{f_{x}}=\frac{(1-\phi)^{-2.5}}{\sqrt{\xi}} f^{\prime \prime}(0, \xi), \\
& \operatorname{Re}_{x}^{-1 / 2} N u_{x}=-\frac{1}{\sqrt{\xi}} \frac{k_{n f}}{k_{f}} \theta^{\prime}(0, \xi), ? \\
& \operatorname{Re}_{x}^{-1 / 2} S h_{x}=-\frac{1}{\sqrt{\xi}} \Phi^{\prime}(0, \xi)
\end{aligned}
$$

\section{SOLUTION METHOD}

We use the spectral local linearization method (SLLM) to solve the system of equations (9)-(11). Eqs. (9) - (11) are linearized using the Gauss-Seidel approach (see Motsa [28]). The principle of the SLLM algorithm is to linearize and decouple the system of equations. Nonetheless, this method has only been used in a limited number of studies, hence its general validation in complex systems remains to be made. The detail derivation of SLLM algorithm is described in [28].

Here, the computational domain in the $\eta$-direction is chosen so that $L=30$. This value was found to give accurate results for all selected physical parameters. Increasing $\eta$ does not change the results to a significant extent. The number of collocation points used in the spectral method discretization is $N_{x}=100$ in all cases. We note that the computation of some quantity, say $F^{n+1}{ }_{r+1}$, at each time step is achieved by iterating using the local linearization method using a known value at the previous time step $n$ as the initial approximation. The calculations were carried out until the desired tolerance level $\epsilon=10^{-6}$ was attained. The tolerance level is the maximum value of the infinity norm of the difference between the values of the calculated quantities, that is to ensure the accuracy of the results, a sufficiently small step size $\Delta \xi$ was used. The step size was chosen to be small enough such that further reduction did not change the results.

\section{RESULTS AND DISCUSSION}

The nanofluid velocity profiles $f^{\prime}(\eta, \xi)$ for different values of the nanoparticle volume fraction $\phi$ and stretching or shrinking parameter $\varepsilon$ are given in Fig. 2. Fig. 2(a) shows that the nanofluid velocity decreases when the nanoparticle volume fraction increases. Fig. 2(b) shows that the $\mathrm{TiO}_{2-}$ water nanofluid has marginally higher values increasing stretching parameter values $\varepsilon>0$ while opposite trend is observed for a shrinking parameter $\varepsilon<0$. The stretching or shrinking is due to the impulsive force that acts in $x$-direction $(+v e$ or $-v e)$. The findings in the case of $C u$-water nanofluid are similar to the result obtained by Grosan and Pop [29].

The unsteady boundary layer flow of two water based nanofluids along a vertical stretching or shrinking cone was studied. The flow was subject to viscous dissipation, internal heat generation and a chemical reaction. The non-similar partial differential equations were solved using the spectral local linearization method. We have investigated the effects of the nanoparticle volume fraction $(\phi)$, magnetic field parameter $(M n)$, buoyancy parameter $(\lambda)$, stretching or shrinking parameter $(\varepsilon)$, heat generation parameter $(\delta)$, Eckert number $(E c)$, chemical reaction parameter $(\gamma)$ on the nanofluid velocity, temperature and concentration profiles as well as the skin friction coefficient, heat and mass transfer coefficients. We have used the values $m=4, \operatorname{Pr}=6.7$ and $S c$ $=1$ unless otherwise stated. We have considered Copper $(\mathrm{Cu})$ and Titanium oxide $\left(\mathrm{TiO}_{2}\right)$ nanoparticles with water as the base fluid. We note that $\epsilon<0$ for a shrinking cone and $\epsilon>0$ indicates that the cone is stretching. The thermophysical properties of the base fluid and the nanoparticles are listed in Table 1. To determine the accuracy of the numerical method, solutions for some special cases are presented in Tables 2 and 3 . The results are in excellent agreement with the work of Kameswaran et al. [3], Wang [22] and Jafar et al. [23].

Table 1. Thermophysical properties of the base fluid and the nanoparticles Oztop and Abu-Nada [25]

\begin{tabular}{|c|c|c|c|c|}
\hline Physical properties & $\begin{array}{c}\mathrm{Cp} \\
(\mathrm{J} / \mathrm{kgK})\end{array}$ & $\begin{array}{c}P \\
\left(\mathrm{Kg} / \mathrm{m}^{3}\right)\end{array}$ & $\begin{array}{c}K \\
(\mathrm{~W} / \mathrm{mK})\end{array}$ & $\begin{array}{c}\beta \\
\times 10^{5} \\
\left(K^{-1}\right)\end{array}$ \\
\hline Pure water $\left(\mathrm{H}_{2} \mathrm{O}\right)$ & 4179 & 997.1 & 0.613 & 21 \\
\hline Copper $(\mathrm{Cu})$ & 385 & 8933 & 401 & 1.67 \\
\hline $\begin{array}{c}\text { Titanium Oxide } \\
\left(\mathrm{TiO}_{2}\right)\end{array}$ & 686.2 & 4250 & 8.9538 & 0.9 \\
\hline
\end{tabular}


Table 2. Comparison of the skin friction coefficient $f$ ' $(0,1)$ and heat transfer rate $-\theta^{\prime}(0,1)$, for various values of stretching or shrinking parameter $\varepsilon$ when $\mathrm{Mn}=\lambda=\delta=\mathrm{Ec}=0, \mathrm{~m}=3, \operatorname{Pr}$ $=1$ and $\phi=0$.

\begin{tabular}{|c|c|c|c|c|}
\hline & \multicolumn{2}{|c|}{ Suali et al. [30] } & \multirow{2}{*}{$\begin{array}{c}\begin{array}{c}\text { Present } \\
\text { results }\end{array} \\
f^{\prime \prime}(0,1) \\
\end{array}$} & \multirow{2}{*}{$\begin{array}{c}\text { SLL Method } \\
-\theta^{\prime}(0,1) \\
\end{array}$} \\
\hline$\epsilon$ & $f^{\prime \prime}(0,1)$ & $-\theta^{\prime}(0,1)$ & & \\
\hline & $\begin{array}{ll}4 & -7.086378\end{array}$ & 2.116738 & -7.086378 & 2.116738 \\
\hline & -4.276545 & 1.870671 & -4.276542 & 1.870671 \\
\hline & $0.2 \quad 1.051130$ & 0.913303 & 1.051130 & 0.913303 \\
\hline & $0.1 \quad 1.146561$ & 0.863452 & 1.146561 & 0.863452 \\
\hline & $-0.2 \quad 1.373886$ & 0.501448 & 1.373886 & 0.698748 \\
\hline & $-0.5 \quad 1.495672$ & 0.501448 & 1.495670 & 0.501447 \\
\hline & -1.151 .082232 & -0.2979953 & 1.082491 & -0.297346 \\
\hline
\end{tabular}

Table 3. Comparison of the skin friction coefficient $f$ ' ' $(0,1)$ for various values of stretching or shrinking parameter $\varepsilon$ when $\mathrm{Mn}=\lambda=0, \mathrm{~m}=3$ and $\phi=0$.

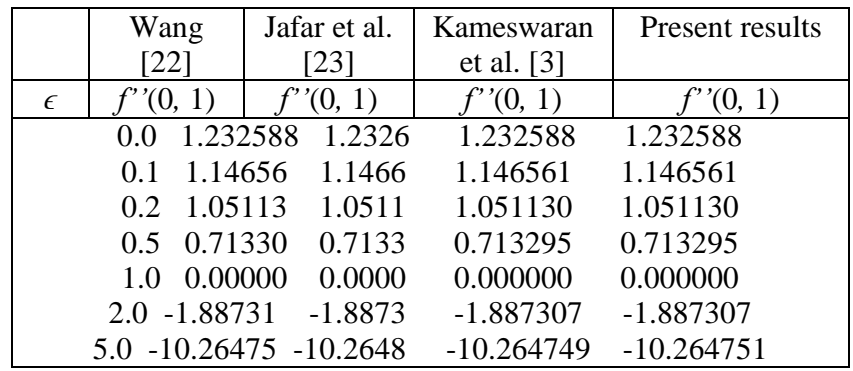

(a)
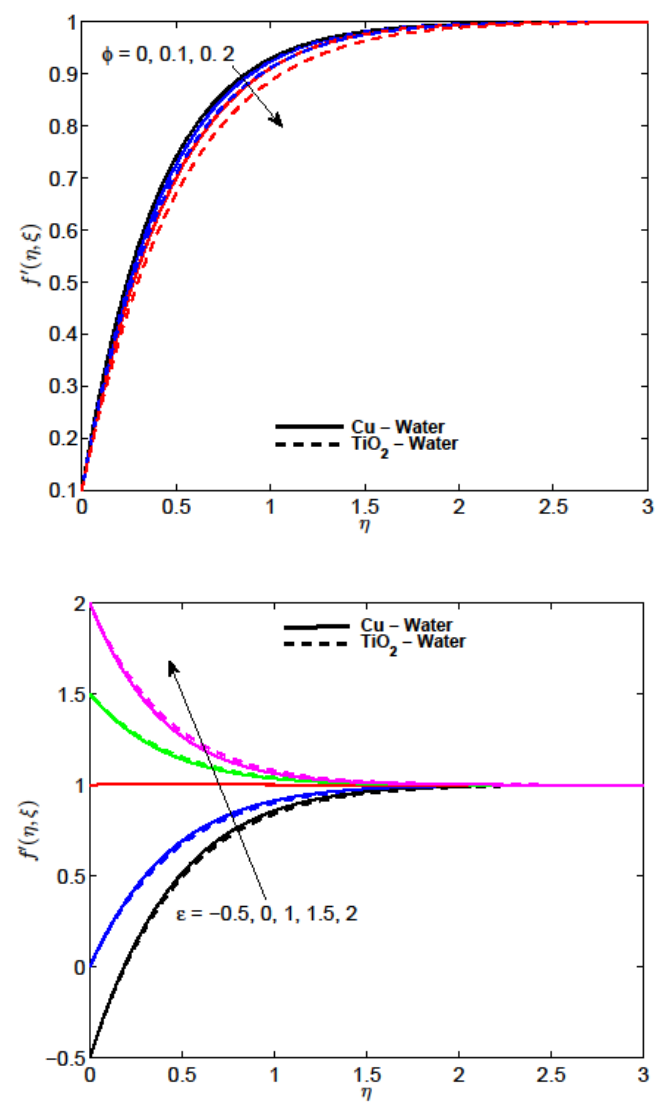

(b)

Figure 2. a) Effect of various nanoparticle volume fraction $(\phi)$. (b) Effect of stretching or shrinking parameter $(\varepsilon)$ on velocity profiles
Figs. 3 and 4 illustrate the effects of nanoparticle volume fraction $(\phi)$, stretching or shrinking parameter $(\varepsilon)$, heat generation parameter $(\delta)$ and Eckert number $(E c)$ on the temperature profiles $\theta(\xi, \eta)$ for both $\mathrm{Cu}$-water and $\mathrm{TiO}_{2}$-water nanofluids. The temperature profiles increase with increases in $\phi, \delta$ and $E c$. We note that an increase in the nanoparticle volume fraction increases the thermal conductivity of the nanofluid significantly, and that internal heat generation increases the temperature of the nanofluid. An increase in the Eckert number increases dissipation due to fluid viscosity or frictional heating. The surface gets cooler when the dissipation increases and as a result there is a transfer of heat from the surface to the nanofluid which causes the temperature to increase. On the other hand, the $C u$-water nanofluid temperature decreases with increasing stretching parameter $\varepsilon>0$ and increases with shrinking parameter $\varepsilon<0$.

(a)
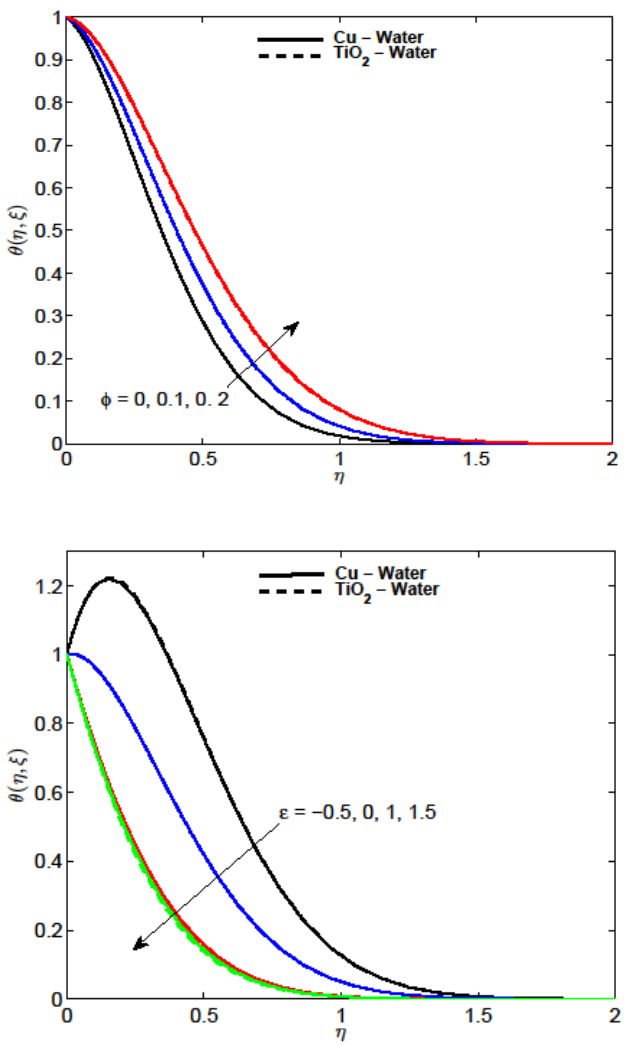

(b)

Figure 3. (a) Effect of nanoparticle volume fraction $(\phi)$. (b) Effect of stretching or shrinking parameter $(\varepsilon)$ on temperature profiles.

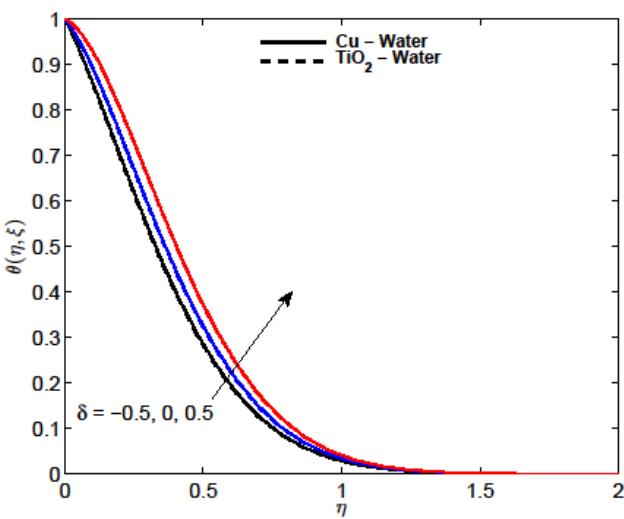


(b)

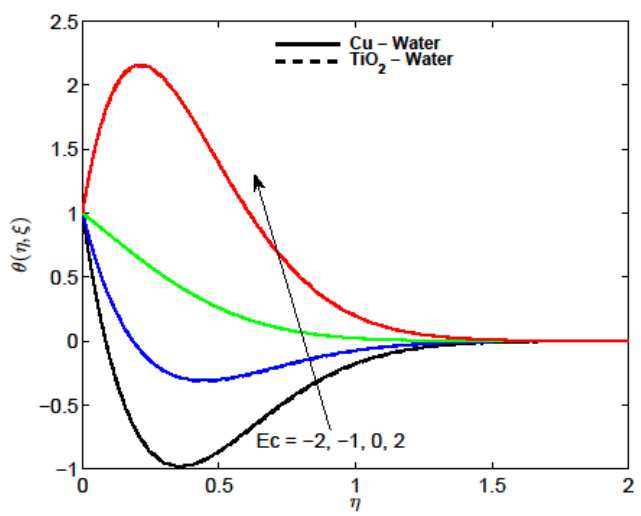

Figure 4. (a) Effect of heat generation parameter $(\delta)$. (b)

Effect of Eckert number $(E c)$ on temperature profiles.

Fig.5 shows that the concentration profiles increase with nanoparticle volume fraction but reduce with stretching. It is observed from Fig. 6 that the concentration profiles decrease with $M n, \lambda$ and $\gamma$ for both nanofluids. It is observed in Fig.7(a) that the $\mathrm{Cu}$-water nanofluid has higher skin friction coefficient values compared to the $\mathrm{TiO}_{2}$-water nanofluid for the increasing values of $\xi$. Fig.7(b) shows that the value of the skin friction coefficient of a $\mathrm{Cu}$-water nanofluid are higher than for $\mathrm{TiO}_{2}$ water nanofluid when $\epsilon>0$ but the opposite trend is observed for $\epsilon<0$. These results show that the skin friction coefficient decreases with increasing nanoparticle volume friction and stretching or shrinking parameters.

(a)
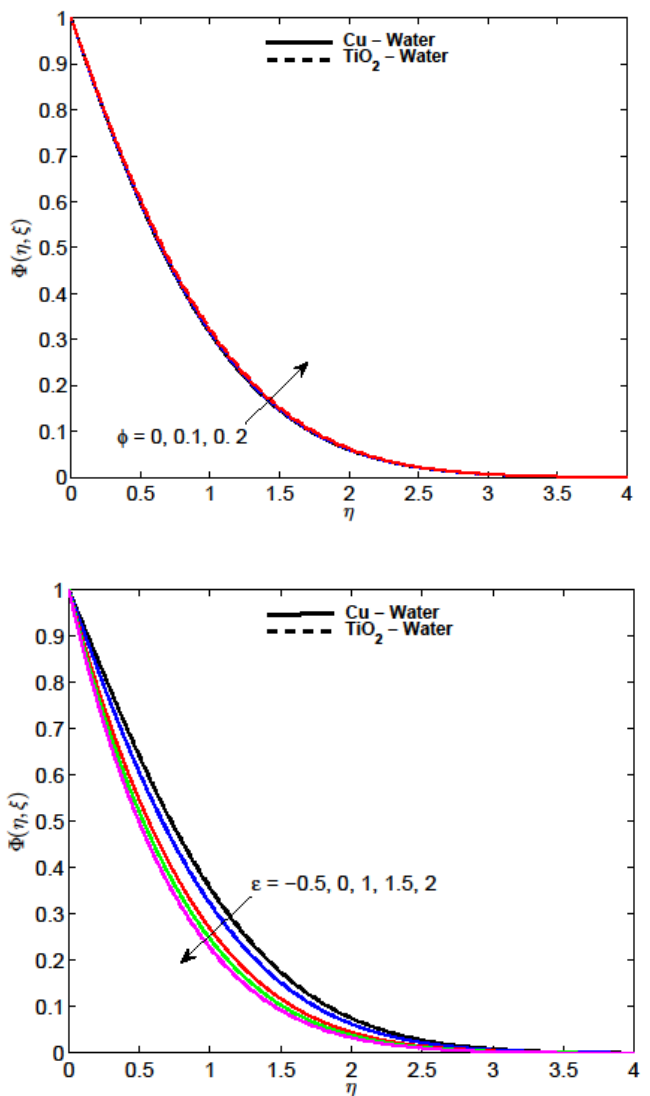

(b)

Figure 5. (a) Effect of nanoparticle volume fraction $(\phi)$. (b) Effect of stretching or shrinking parameter $(\varepsilon)$ on concentration profiles

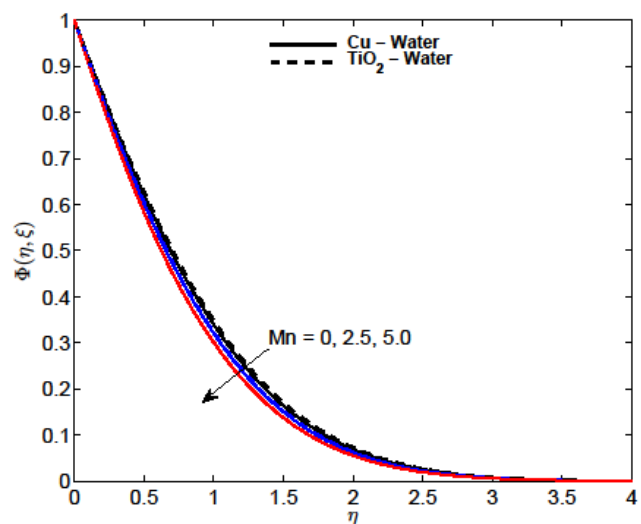

(a)

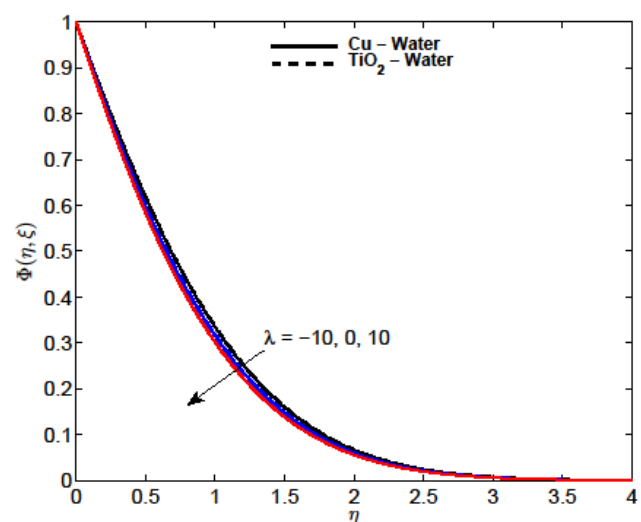

(b)

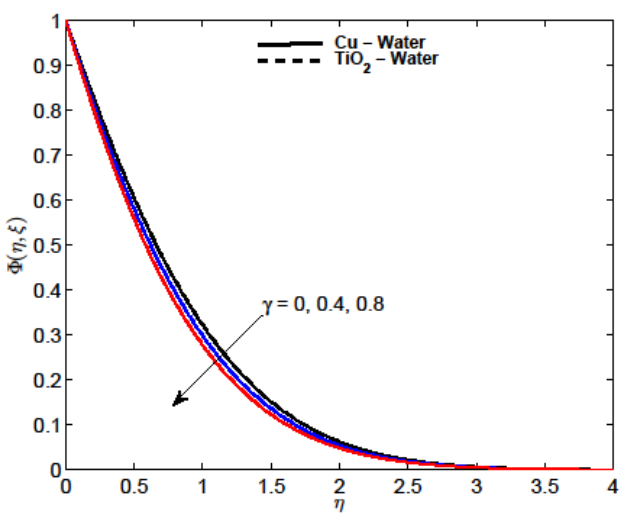

(c)

Figure 6. a) Effect of magnetic field parameter (Mn), (b) Effect of buoyancy parameter $(\lambda)$, (c) Effect of chemical reaction parameter $(\gamma)$ on concentration profiles.

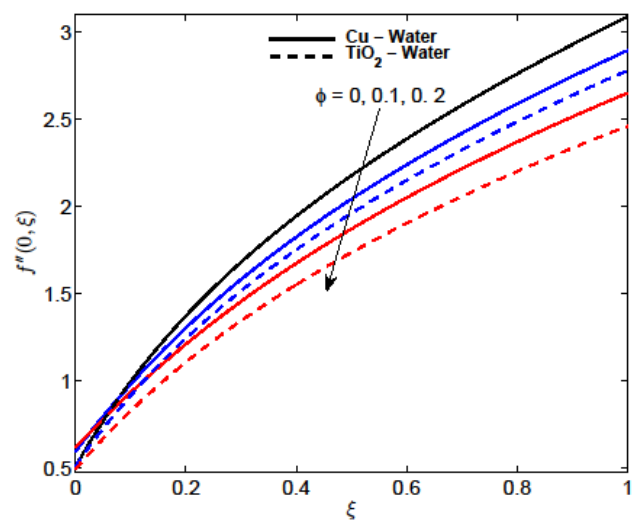


(b)

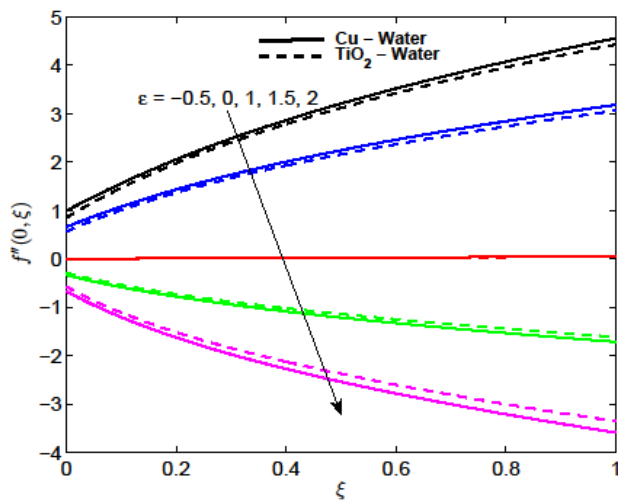

Figure 7. (a) Effect of nanoparticle volume fraction $(\phi)$. (b) Effect of stretching or shrinking parameter $(\varepsilon)$ on skin friction coefficients.

Fig. 8(a) shows that the heat transfer rate for a $\mathrm{Cu}$-water nanofluid is smaller compared to the $\mathrm{TiO}_{2}$-water nanofluid at the surface of the cone as the nanoparticle volume friction increases. We observe from Figs. 8 (a) and (b) that the heat transfer rate decreases with the increasing $\phi$ but the opposite trend is observed for $\epsilon$. The $\mathrm{Cu}$-water gives a smaller heat transfer rate in comparison to the $\mathrm{TiO}_{2}$-water nanofluid for increasing $\delta$. Again, we note that $\delta, E c$ reduce the heat transfer rate in both Figs. 9 (a) and (b).

(a)

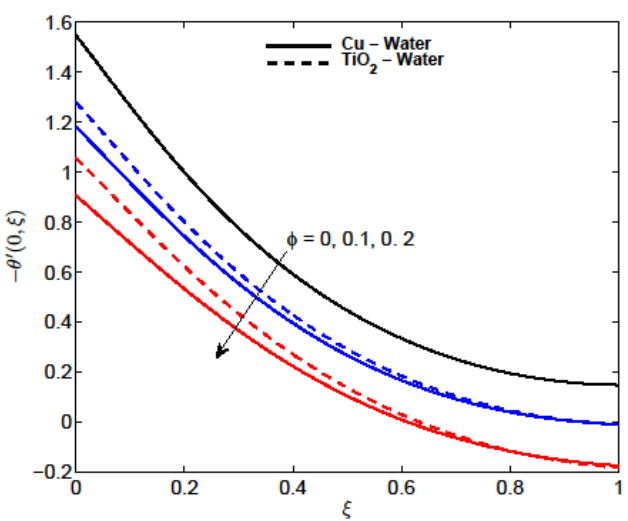

(b)

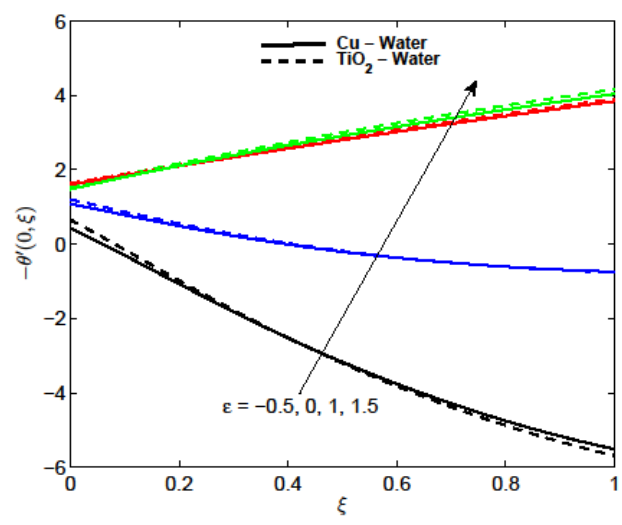

Figure 8. (a) Effect of nanoparticle volume fraction $(\phi)$. (b) Effect of stretching or shrinking parameter $(\varepsilon)$ on heat transfer rate

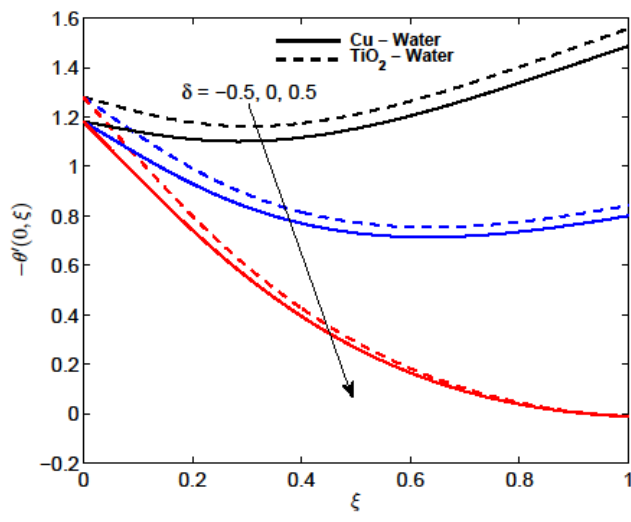

(a)

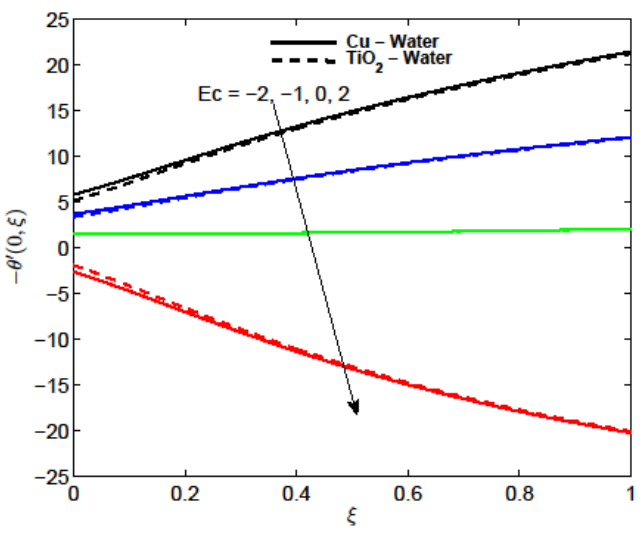

(b)

Figure 9. (a) Effect of heat generation parameter $(\delta)$. (b) Effect of Eckert number $(E c)$ on heat transfer rate.

(a)
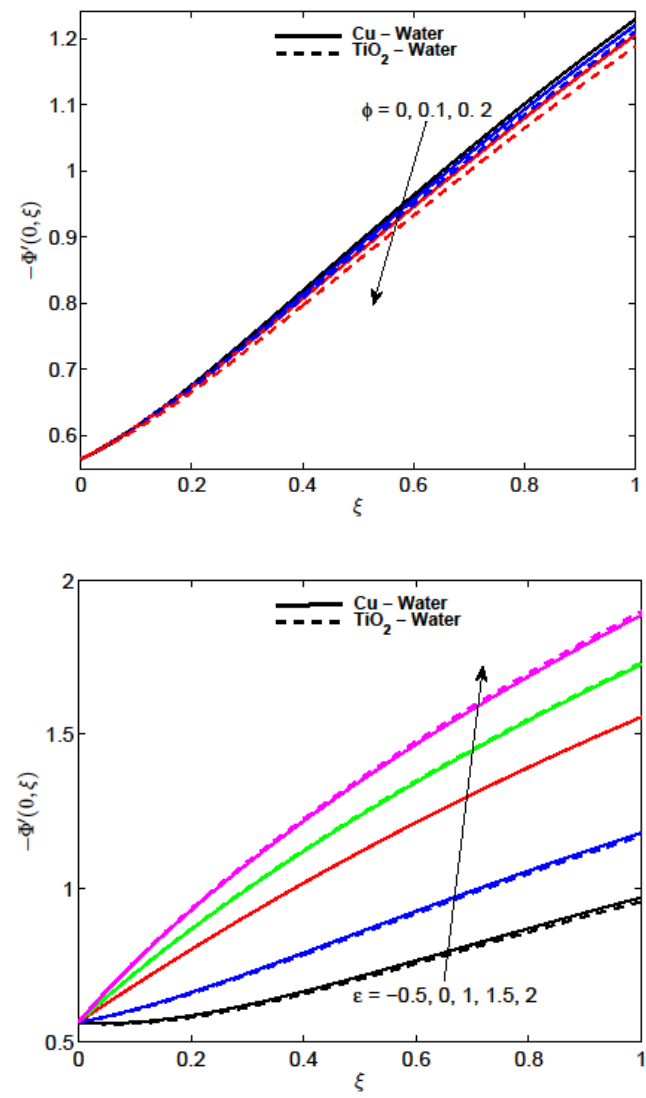

(b)

Figure 10. (a) Effect of nanoparticle volume fraction $(\phi)$. (b) Effect of stretching or shrinking parameter $(\varepsilon)$ on mass transfer rate 
The impact of increasing the nanoparticle volume fraction, stretching or shrinking parameters, magnetic field, buoyancy force parameter and chemical reaction parameters on the mass transfer rate at the cone surface is shown in Fig. 10. Fig. 10 (a) shows that the $\mathrm{Cu}$-water nanofluid takes higher values than the $\mathrm{TiO}_{2}$-water nanofluid for the increasing value of $\phi$. The mass transfer rate reduces with increasing $\phi$ for both nano fluids but the opposite trend is observed in Fig. 10 (b).

(a)
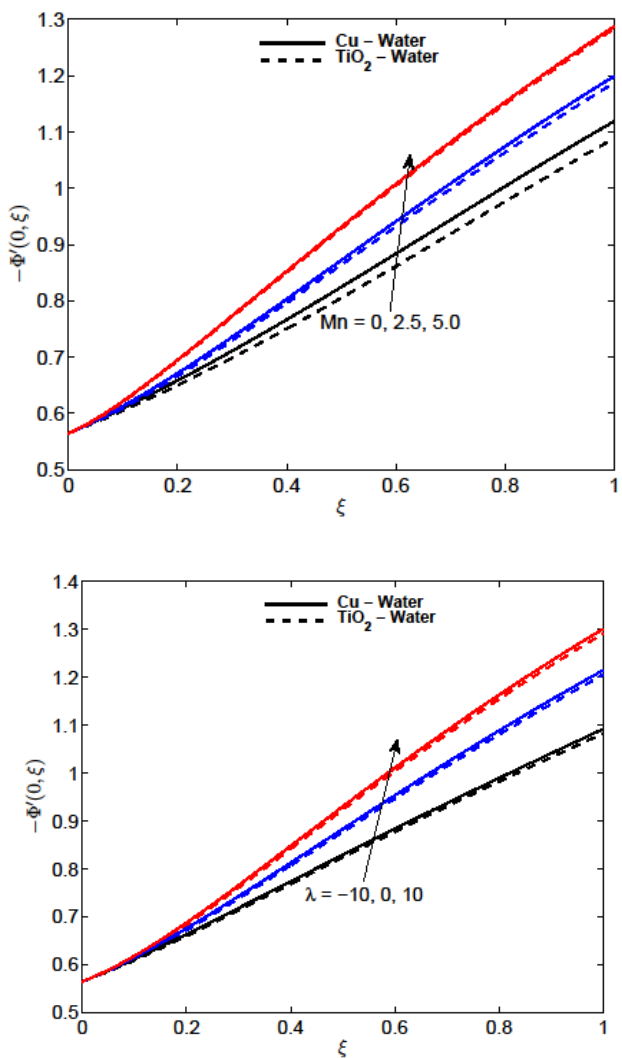

(b)

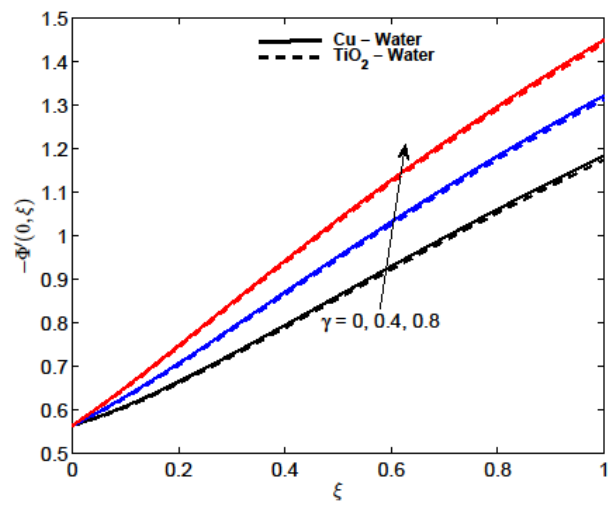

(c)

Figure 11. (a) Effect of magnetic field parameter (Mn), (b) Effect of buoyancy parameter $(\lambda)$, (c) Effect of chemical reaction parameter $(\gamma)$ on mass transfer rate.

Fig. 11 shows that the mass transfer rate increases with increasing $M n, \lambda$ and $\gamma$ for both nanofluids. The effects of magnetic field parameter $M n$ and buoyancy force parameter $\lambda$ on the velocity and the skin friction coefficient are shown in Figs. 12 and 13 respectively. We note that the $\mathrm{Cu}$-water nanofluid assumes higher velocity and skin friction coefficient than the $\mathrm{TiO}_{2}$-water nanofluid for the increasing values of $M n, \lambda$.

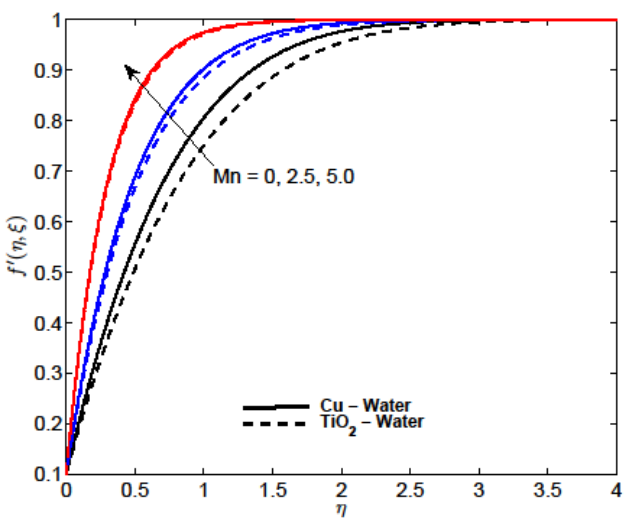

(a)

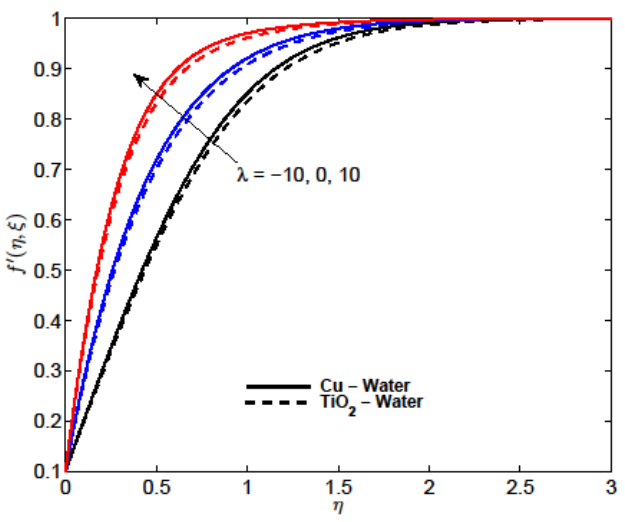

(b)

Figure 12. a) Effect of magnetic field parameter (Mn), (b) Effect of buoyancy parameter $(\lambda)$ on velocity profiles.

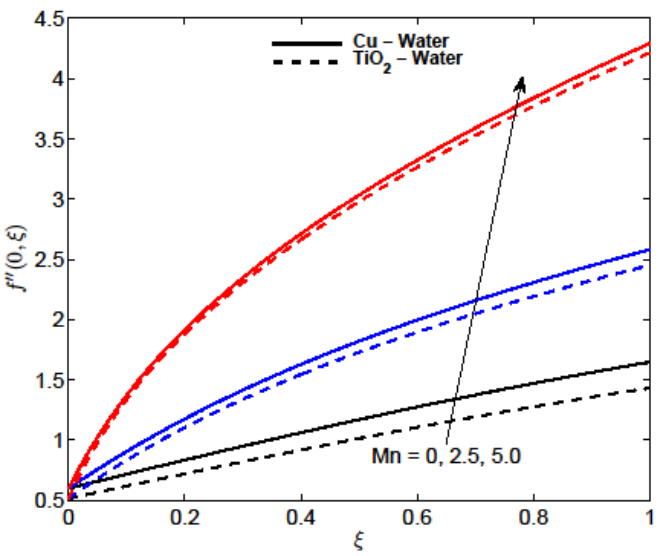

(a)

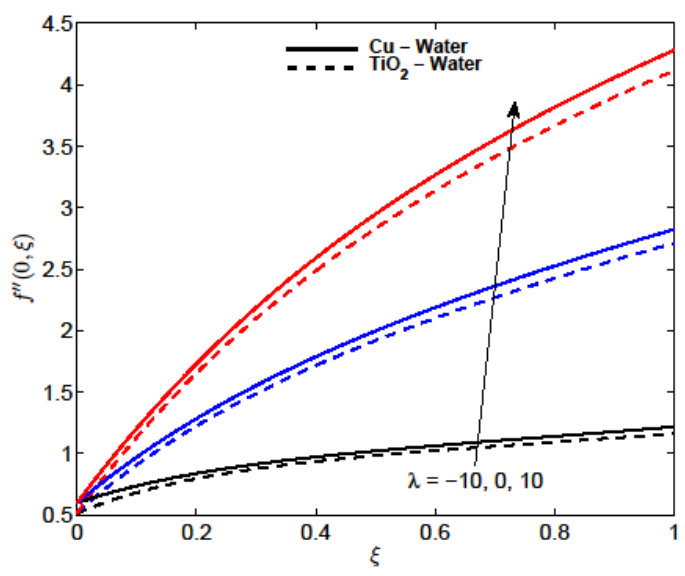

(b)

Figure 13. (a) Effect of magnetic field parameter (Mn), (b) Effect of buoyancy parameter $(\lambda)$ on skin friction coefficients. 
The influence of the magnetic field and buoyancy force parameters on the nanofluid temperature and the local Nusselt number are captured in Figs. 14 and 15, respectively. It is noted from Fig. 15 that a $\mathrm{Cu}$-water nanofluid has smaller values of the local Nusselt number than a $\mathrm{TiO}_{2}$-water nanofluid for the increasing value of $M n, \lambda$. We further observe that the local Nusselt number decreases with increases in $M n$ and $\lambda$.

(a)
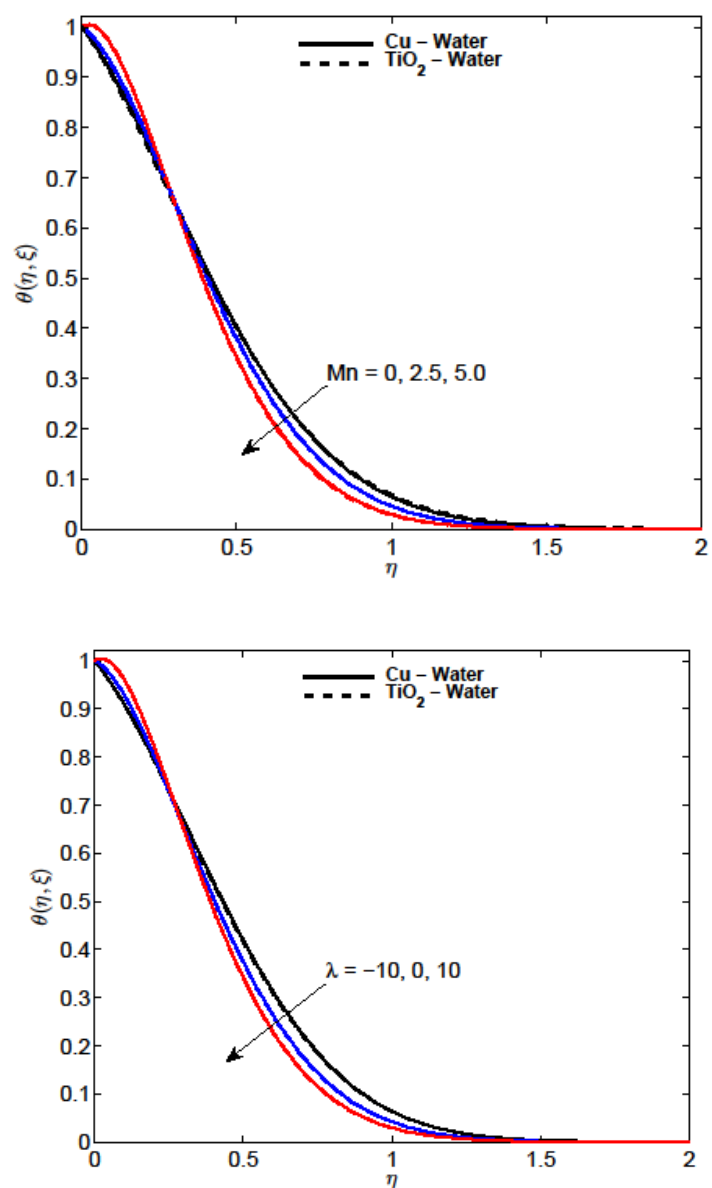

(b)

Figure 14. (a) Effect of magnetic field parameter (Mn), (b) Effect of buoyancy parameter $(\lambda)$ on temperature profiles

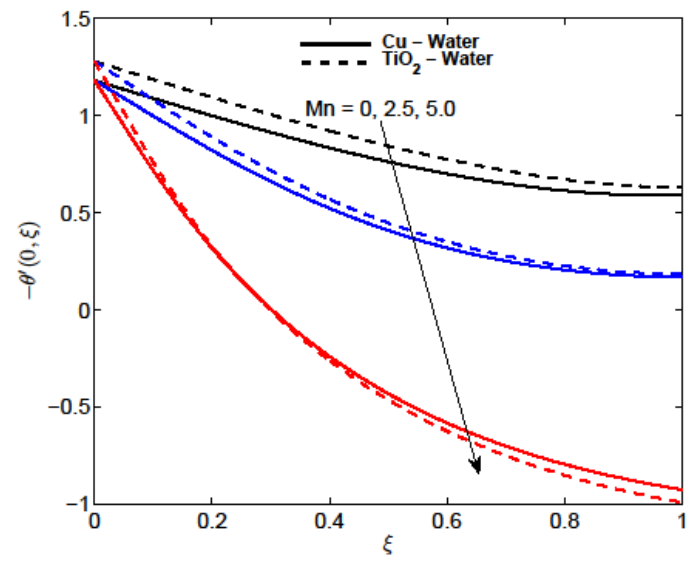

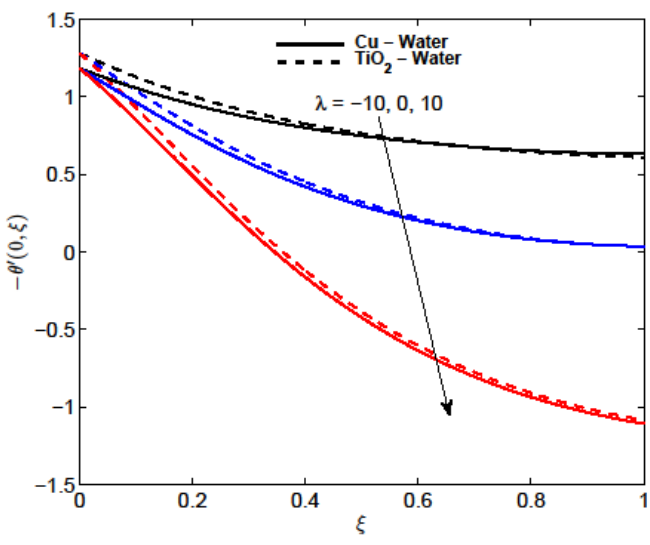

(b)

Figure 15. (a) Effect of magnetic field parameter (Mn), (b) Effect of buoyancy parameter $(\lambda)$ on heat transfer rate.

Fig. 16 shows the effect of the Prandtl number on the temperature profiles. The temperature profiles and the thermal boundary layer thickness quickly decreases with increasing Prandtl numbers. The Prandtl number is a means to increase fluid viscosity resulting in a reduction in the flow velocity and temperature. Here, the thermal boundary layer thickness decreases with increasing Prandtl number, which is consistent with the findings of various researchers. Fig 17 shows the streamlines for different value of $\epsilon$ when the other values are fixed.

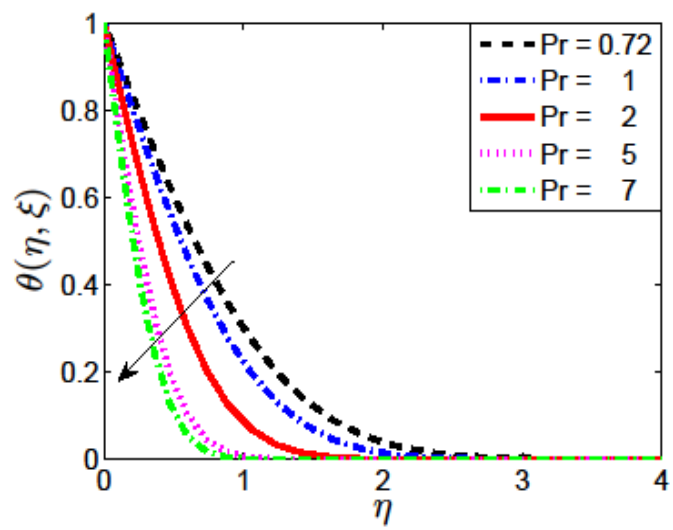

Figure 16. Effect of $(\mathrm{Pr})$ on temperature profiles

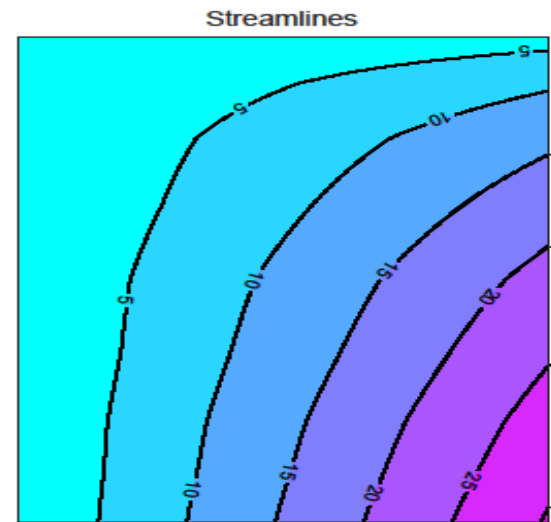

(a) 


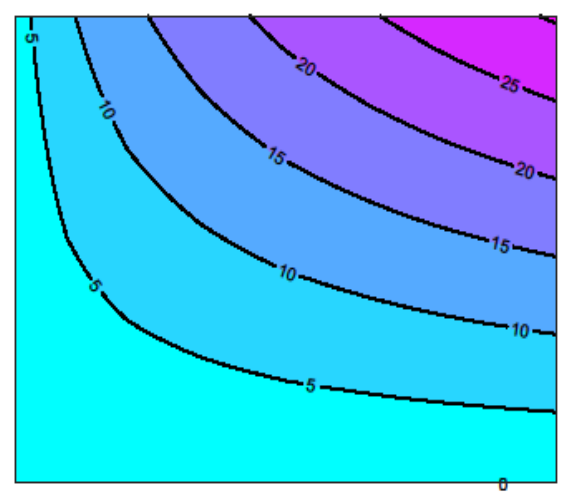

b)

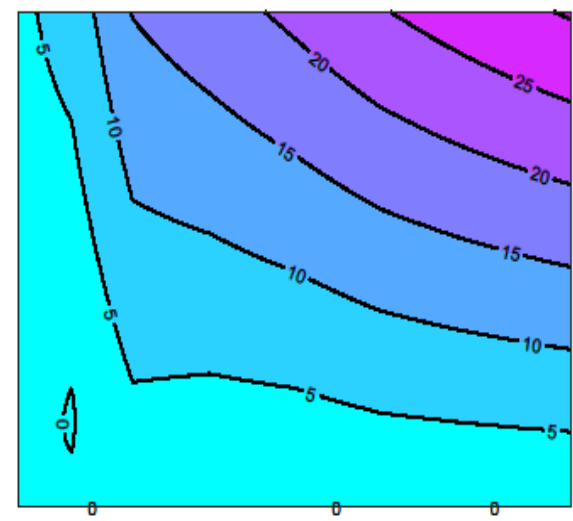

(c)

Figure 17. Streamlines for (a) $\epsilon=-1.2$, (b) $\epsilon=1.0$, (c) $\epsilon=$ 2.0 when the other parameters are fixed.

\section{CONCLUSIONS}

The unsteady boundary layer flow of a viscous, incompressible fluid along a vertical stretching or shrinking cone was investigated. The effects of viscous dissipation, internal heat generation and a chemically reactive species have been taken into account for $\mathrm{Cu}$-water and $\mathrm{TiO} 2$-water nanofluids.

It was found that the viscous dissipation has the effect of increasing the nanofluid temperature within the boundary layer region while the rate of heat transfer from the surface decreases with an increase in viscous dissipation. The internal heat generation has the tendency to increase the nanofluid temperature and reduce the rate of heat transfer at the surface of the cone. The nanoparticle concentration decreases while the wall mass transfer rate increases with the increase in the strength of a chemical reaction.

\section{ACKNOWLEDGEMENTS}

All the authors are thankful to University of KwaZuluNatal, South Africa and Amity University, Kolkata, India for the necessary support.

\section{REFERENCES}

[1] Choi S.U.S., Eastman J.A. (1995). Enhancing thermal conductivity of fluids with nanoparticles, San
Francisco, ASME International Mechanical Engineering Congress and Exposition, Vol. 231, pp. 99-103.

[2] Kameswaran P.K., Narayana M., Sibanda P., Murthy P.V.S.N. (2012). Hydromagnetic nanofluid flow due to a stretching or shrinking sheet with viscous dissipation and chemical reaction effects, International Journal of Heat and Mass Transfer, Vol. 55, pp. 7587-7595. DOI: 10.1016/j.ijheatmasstransfer.2012.07.065

[3] Kameswaran P.K., Sibanda P., Ramreddy C., Murthy P.V.S.N. (2013). Dual solutions of stagnation-point flow of a nanofluid over a stretching surface, Boundary Value Problems, Vol. 188, pp. 1-12. DOI: 10.1186/1687-2770-2013-188

[4] Fauzi E.L.A., Ahmad S., Pop I. (2012). Mixed convection boundary layer flow from a vertical cone in a porous medium filled with a nanofluid, World Academy of Science, Engineering and Technology, Vol. 6, pp. 15-18. DOI: scholar.waset.org/1307$\underline{6892 / 13549}$

[5] Boutra A., Ragui K., Labsi N., Benkahla Y.K. (2017). Free convection enhancement within a nanofluid' filled enclosure with square heaters, International Journal of Heat and Technology, Vol. 35, pp. 447-458. DOI: 10.18280/ijht.350302

[6] Ambethkar V., Kumar M. (2017). Numerical solutions of 2-D unsteady incompressible flow with heat transfer in a driven square cavity using streamfunctionvorticityformulation, International Journal of Heat and Technology, Vol. 35, pp. 459-473. DOI: 10.18280/ijht.350303

[7] Cheng C. (2012). Natural convection boundary layer flow over a truncated cone in a porous medium saturated by a nanofluid, Int. Commun. Heat Mass Transf. Vol. 39, pp. 231-235. DOI: 10.1016/j.icheatmasstransfer.2011.11.002

[8] Chamkha A.J., Abbasbandy S., Rashad A.M., Vajravelu K. (2013). Radiation effects on mixed convection about a cone embedded in a porous medium filled with a nanofluid, Meccanica, Vol. 48, pp. 275-285. DOI: $10.1007 / \mathrm{s} 11012-012-9599-1$

[9] Nadeem S., Saleem S. (2014). Unsteady mixed convection flow of nanofluid on a rotating cone with magnetic field, Appl. Nanosci., Vol. 4, pp. 405-414. DOI: $10.1007 / \mathrm{s} 13204-013-0213-1$

[10] Hering R.G., Grosh R.J. (1963). Laminar combined convection from a rotating cone, ASME J. Heat Transfer 85, pp. 29-34. DOI: $10.1115 / 1.3614336$

[11] Tien C.L., Tsuji I.J. (1965). A theoretical analysis of laminar forced flow and heat transfer about a rotating cone, ASME J. Heat Transfer, Vol. 87, pp. 184-190. DOI: $10.1115 / 1.3689069$

[12] Koh J.C.Y., Price J.F. (1967). Non-similar boundary layer heat transfer of a rotating cone in forced flow, ASME J. Heat Transfer, Vol. 89, pp. 139-145. DOI: $\underline{10.1115 / 1.3614336}$

[13] Takhar H.S., Chamkha A.J., Nath G. (2004). Effect of thermophysical quantities on the natural convection flow of gases over a vertical cone, International Journal of Engineering Science, Vol. 42, pp. 243-256. DOI: $10.1016 /$ j.ijengsci.2003.07.005

[14] Turkyilmazoglu M. (2009). On the purely analytic computation of laminar boundary layer flow over a rotating cone, International Journal of Engineering 
Science, Vol. 47, pp. 875-882. DOI: 10.1016/j.ijengsci.2009.05.008

[15] Sivaraj R., Kumar B.R. (2003). Viscoelastic fluid flow over a moving vertical cone and flat plate with variable electric conductivity, International Journal of Heat and Mass Transfer, Vol. 61, pp. 119-128. DOI: 10.1016/j.ijheatmasstransfer.2013.01.060

[16] Srinivasa A.H., Eswara A.T. (2013). Unsteady free convection flow and heat transfer from an isothermal truncated cone with variable viscosity, International Journal of Heat and Mass Transfer, Vol. 57, pp. 411420. DOI: 10.1016/j.ijheatmasstransfer.2012.10.054

[17] Roy S., Datta P., Mahanti N.C. (2007). Non-similar solution of an unsteady mixed convection flow over a vertical cone with suction or injection, International Journal of Heat and Mass Transfer, Vol. 50, pp. 181187. DOI: $10.1016 /$ j.ijheatmasstransfer.2006.06.024

[18] Crane L.J. (1970). Flow past a stretching plate, Zeit. Angew. Math. Phys., Vol. 21, pp. 645-647. DOI: 10.1007/BF01587695

[19] Mahapatra T.R., Pal D., Mondal S. (2012). Heat transfer due to Magnetohydrodynamic stagnation-point flow of a Power-law fluid towards a stretching surface in the presence of thermal radiation and suction/injection, ISRN Thermodynamics, Article ID 465864, pp. 1-9, DOI: $10.5402 / 2012 / 465864$

[20] Salem A.M., Abd El-Aziz M. (2008). Effect of Hall currents and chemical reaction on hydromagnetic flow of a stretching vertical surface with internal heat generation/absorption, Applied Mathematical Modelling, Vol. 32, pp. 1236-1254. DOI: 10.1016/j.apm.2007.03.008

[21] Noor N.F.M., Kechil S.A., Hashim I. (2010). Simple non-perturbative solution for MHD viscous flow due to a shrinking sheet, Commun. Nonlinear Sci. Numer. Simul., Vol. 15, pp. 144-148. DOI: $\underline{10.1016 / j . c n s n s .2009 .03 .034}$
[22] Wang C.Y. (2008). Stagnation flow towards a shrinking sheet, Int. J. Non-Linear Mech., Vol. 43, pp. 377-382. DOI: 10.1016/j.ijnonlinmec.2007.12.021

[23] Jafar K., Nazar R., Ishak A., Pop I. (2012). MHD flow and heat transfer over stretching/shrinking sheets with external magnetic field, viscous dissipation and joule effects, Can. J. Chem. Eng., Vol. 90, pp. 1336-1346. DOI: $10.1002 /$ cjce. 20609

[24] Schlichting H., Gersten K. (2003). Boundary Layer Theory, $8^{\text {th }}$ edition, Springer, pp. 86-89. DOI: 10.1007/978-3-662-52919-5

[25] Oztop H.F., Abu-Nada E. (2008). Numerical study of natural convection in partially heated rectangular enclosures filled with nanofluids, Int. J. Heat Fluid Flow, Vol. 29, pp. 1326-1336. DOI: 10.1016/j.ijheatfluidflow.2008.04.009

[26] Gangadhar K., RangaRao T., Subhakar M.J., Sekhar T.V.S. (2014). MHD and mixed convection flow of Maxwell fluid on heat transfer near a stagnation point flow, IOSR Journal of Mathematics, Vol. 10, No. 2, pp. 60-68. DOI: $10.9790 / 5728-10216068$

[27] Mahdy A. (2012). Unsteady mixed convection boundary layer flow and heat transfer of nanofluids due to stretching sheet, Nuclear Engineering and Design, Vol. 249, pp. 248-255. DOI: 10.1016/j.nucengdes.2012.03.025

[28] Motsa S.S. (2013). A new spectral local linearization method for nonlinear boundary layer flow problems, $J$. Appl. Math., 2013, pp. 1-15. DOI: $10.1155 / 2013 / 423628$

[29] Grosan T., Pop T. (2011). Axisymmetric mixed convection boundary layer flow past a vertical cylinder in a nanofluid, International Journal of Heat and Mass Transfer, Vol. 54, pp. 3139-3145. DOI: 10.1016/j.ijheatmasstransfer.2011.04.018

[30] Suali M., Long N.M.A.N., Ishak A. (2012). Unsteady stagnation point flow and heat transfer over a stretching/shrinking sheet with prescribed surface heat flux, App. Math. and Comp. Intel., Vol. 1, pp. 1-11. 